\title{
Crossmodal action selection: Evidence from dual-task compatibility
}

\author{
LyNn HuestegGe AND IRING KoCH \\ RWTH Aachen University, Aachen, Germany
}

\begin{abstract}
Response-related mechanisms of multitasking were studied by analyzing simultaneous processing of responses in different modalities (i.e., crossmodal action). Participants responded to a single auditory stimulus with a saccade, a manual response (single-task conditions), or both (dual-task condition). We used a spatially incompatible stimulus-response mapping for one task, but not for the other. Critically, inverting these mappings varied temporal task overlap in dual-task conditions while keeping spatial incompatibility across responses constant. Unlike previous paradigms, temporal task overlap was manipulated without utilizing sequential stimulus presentation, which might induce strategic serial processing. The results revealed dual-task costs, but these were not affected by an increase of temporal task overlap. This finding is evidence for parallel response selection in multitasking. We propose that crossmodal action is processed by a central mapping-selection mechanism in working memory and that the dual-task costs are mainly caused by mapping-related crosstalk.
\end{abstract}

Numerous studies have demonstrated that multitasking leads to poorer performance than does executing one task (e.g., Pashler, 1994). Typically, multitasking research focuses either on manipulating the temporal relation of two tasks by systematically varying their temporal overlap via the psychological refractory period (PRP) paradigm (Welford, 1952; for a review, see Pashler, 1994) or on comparing single-task performance with performance in trials where the stimuli for both tasks are presented at the same time via the dual-task paradigm (e.g., Navon \& Miller, 1987). The present study combines both approaches. We utilized a single stimulus for both tasks in order to gain suitable control over stimulus-related processes and varied the spatial stimulus-response (S-R) mappings across tasks in order to manipulate temporal task overlap. Using this novel experimental approach yielded evidence for parallel response selection in multitasking.

In the PRP paradigm, the manipulation of stimulus onset asynchrony (SOA) between tasks induces a systematic variation of temporal task overlap. As a result, more overlap (i.e., shorter SOAs) leads to a prolongation of response times (RTs) in the second task, but not in the first task (i.e., the PRP effect; Welford, 1952). More specifically, at short SOAs, every further decrease of SOA is associated with an almost equal increase of RTs in the second task.

The PRP effect has been explained in terms of a central response-selection bottleneck in cognitive processing (Pashler, 1994). Within the bottleneck model, task processing is conceived of as a series of distinct stages, such as perceptual processing, response selection, and response execution. Empirical evidence from numerous PRP studies involving the experimental manipulation of the vari- ous processing stages in both tasks suggests that primarily, the response-selection stage acts as a central bottleneck. More specifically, according to such an account, response selection must be devoted to only one response at a time, so that processing of the second task is suspended until response selection in the first task has been finished (Pashler, 1994). This serial central processing account thus serves as a straightforward explanation of the PRP effect. The PRP effect occurs even when sensory and motor modalities are distinct for the two tasks (e.g., an auditory-manual Task 1 and a visual-vocal Task 2), suggesting that the central response-selection stage is basically an amodal origin of the dual-task costs (e.g., Marois \& Ivanoff, 2005; but see Hazeltine, Ruthruff, \& Remington, 2006). More recent models of multitasking extended the central bottleneck model to account for more complex multitasking situations (e.g., Byrne \& Anderson, 2001; Salvucci \& Taatgen, 2008), but the core idea of serial central processing remained untouched.

A major advantage of the PRP paradigm is the possibility of manipulating temporal task overlap to study the mechanisms of multitasking. However, it was argued that the PRP effect might specifically arise due to the successive stimulus presentation, suggesting strategic serial response processing to the participants (see Meyer \& Kieras, 1997). This possibility might compromise any generalization to settings where stimuli are not separated by a given time lag; thus, the PRP paradigm is not suited to finally answering the question of whether response selection can be accomplished in parallel. Furthermore, the PRP paradigm captures only a portion of performance costs in multitasking. For example, PRP studies that ad- 
ditionally included single-task controls demonstrated that RT1 and RT2 in PRP trials were longer (at any SOA) than the respective single-task RTs (e.g., Herman \& Kantowitz, 1970; Hommel, 1998; Pashler, 1984), suggesting further sources of performance costs in multitasking beyond central response-selection bottlenecks.

By comparison, in the dual-task paradigm, dual-task costs are typically assessed by subtracting RTs in singletask conditions from RTs in dual-task conditions (e.g., Navon \& Miller, 1987). A major advantage of the dual-task paradigm is that the simultaneous presentation of stimuli in dual-task conditions does not suggest a serial responseplanning strategy to the participants. A major drawback, however, is that this procedure does not allow one to manipulate temporal task overlap, thus limiting the scope regarding the analysis of underlying mechanisms of multitasking. Additionally, whereas some portion of the dual-task costs in the dual-task paradigm might be specifically related to central bottlenecks or central resource limitations (similar to costs in the PRP paradigm), some other portion appears to be due to other factors, such as crosstalk (see below) or more general concurrence costs (e.g., expectancies) of performing two tasks at a time (see Logan \& Gordon, 2001). For example, Gottsdanker (1979) demonstrated that RTs were slowed substantially when the stimulus of a secondary task was expected but did not appear. Thus, the dual-task paradigm does not allow one to separately assess the various sources of performance costs in multitasking.

Similar to the PRP effect, performance costs in the dualtask paradigm are also often more pronounced in one task than in the other (e.g., Fagot \& Pashler, 1992; Navon \& Miller, 1987). A possible account of such differences in performance costs can be built upon the assumption that single-task processing speed determines the pattern of performance costs in multitasking. This assumption was first developed within the context of the Stroop effect (Posner $\&$ Snyder, 1975; Stroop, 1935; but for a critical discussion, see MacLeod, 1991). More specifically, the effects of single-task processing speed on dual-task performance in the dual-task paradigm can be derived within the central bottleneck framework (Pashler, 1994). Similar single-task processing speeds of two tasks implies larger temporal task overlap in the dual-task situation and should thus increase the amount of temporal overlap of response-selection stages (everything else being equal). When we assume that the system schedules the slower task to come second (i.e., because this speeds overall completion time, measured across both tasks), this should lead to a postponement of response selection in the slower task, resulting in greater dual-task costs than in a situation with smaller temporal task overlap. In the latter, response selection in the faster task should, in many cases, already be completed before processing in the slower task enters the bottleneck, minimizing the potential for dual-task costs. In sum, the central bottleneck account predicts substantially greater dual-task costs in the dual-task paradigm when both tasks show similar single-task RTs (equivalent to relatively larger temporal task overlap in dual-task conditions), as compared with dissimilar single-task RTs (equivalent to relatively smaller temporal task overlap in dual-task conditions).
Alternatively, more recent accounts of dual-task costs assume that central processing of both tasks can principally be accomplished in parallel (e.g., Meyer \& Kieras, 1997; Navon \& Miller, 2002; Tombu \& Jolicœur, 2003), attributing the typical PRP effect to the specific situation of sequential stimulus presentation in the PRP paradigm. According to these accounts, dual-task costs are due to strategic serial processing of tasks (as in the PRP paradigm) or to another source of dual-task interference that may play a role during parallel processing of responses. More specifically, whenever two tasks with dimensional overlap (Kornblum, Hasbroucq, \& Osman, 1990) are processed (e.g., when both tasks involve a left/right dimension), this leaves room for crosstalk to occur (Huestegge \& Koch, 2009; Koch, 2009; Navon \& Miller, 1987). Contrary to the serial bottleneck account, a parallel crosstalk account would not necessarily predict pronounced differences in dual-task costs as a function of temporal task overlap in the dual-task paradigm, as long as the source of crosstalk (e.g., spatially incompatible responses) within a trial remains constant.

The present study was conducted to directly test these alternative accounts (serial vs. parallel) of responserelated processes in multitasking by combining the strengths while avoiding the major drawbacks of both the PRP paradigm and the classic dual-task paradigm. To gain suitable control over stimulus-related processes, we implemented a single-onset paradigm, in which two responses were triggered at the same time and by a common stimulus (Fagot \& Pashler, 1992). By using only one stimulus for both tasks, we ensured that the perceptual analysis of the task-relevant feature would be the same for both tasks and thus would be completed for both tasks at the same time. To manipulate temporal task overlap in the dual-task conditions, we systematically varied the duration of the response-selection stages via different spatial S-R mappings (e.g., Sanders, 1998) in the individual tasks. In the context of dual-task settings with two spatial tasks, the introduction of S-R incompatibility in one task (but not in the other) leads to cross-task response incompatibility (Lien \& Proctor, 2002). Crucially, this manipulation offers the opportunity to introduce a comparable amount of prolongation of the response-selection stage in each of the two tasks. Given the assumed amodal nature of response selection (e.g., Marois \& Ivanoff, 2005; but see Hazeltine et al., 2006), inversely implementing S-R incompatibility in either of the two tasks should affect response selection to a similar extent, while the overall cross-response incompatibility remains constant (for similar manipulations in another context, see Duncan, 1979; Vu \& Proctor, 2006). More specifically, in one condition, S-R mappings were spatially compatible in Task 1 but incompatible in Task 2 , whereas, in an alternative condition, S-R mappings were incompatible in Task 1 but compatible in Task 2. Such an inverted response incompatibility paradigm allows us to manipulate the difference in single-task processing duration while keeping the overall potential for crosstalk (in terms of spatial response-response incompatibility) constant.

As a prerequisite for a successful manipulation of temporal task overlap within this paradigm, we utilized two 
response modalities with highly dissimilar overall RT levels - namely, saccades and manual responses. Typically, saccades are executed much faster than manual responses (e.g., Mather \& Fisk, 1985; Pashler, Carrier, \& Hoffman, 1993). The simultaneous execution of a compatible saccade and an incompatible manual response should amplify the a priori RT difference between these modalities (equivalent to relatively small temporal task overlap in dual-task trials), whereas the simultaneous execution of an incompatible saccade and a compatible manual response should substantially assimilate the RT levels of the response modalities (equivalent to relatively larger temporal task overlap in dual-task trials). More specifically, in the condition with smaller temporal task overlap, participants responded to a single lateralized auditory stimulus with a spatially compatible saccade to a prespecified location on the screen and/or with an incompatible manual response. In the condition with larger temporal task overlap, this setting was reversed, so that participants responded with a spatially incompatible saccade but with a compatible manual response. We used auditory stimuli to ensure comparable sensorimotor transformation processes for both responses (similar levels of input-output modality compatibility; see Hazeltine et al., 2006).

Unlike previous paradigms, the present procedure can be used to determine different sources of dual-task costs (i.e., central bottleneck and crosstalk) separately. A comparison of dual-task costs across temporal task overlap conditions should allow us to assess whether larger temporal task overlap leads to an increase of dual-task costs, as is predicted by serial bottleneck accounts. The contribution of crosstalk to performance costs should be reflected mainly in the comparison between single-task and dual-task performance. A more elaborate model of the specific crosstalk mechanism at work will be presented in the Discussion.

In a recent series of experiments that also utilized the single-onset paradigm in the study of simultaneously executed saccades and manual responses toward auditory stimuli, we demonstrated that dual-task costs occurred even when both responses were spatially compatible (Huestegge \& Koch, 2009). Additionally, we demonstrated that dual-task costs substantially increased when the potential for crosstalk between responses was enhanced (e.g., by introducing crossed-hands conditions), suggesting that crosstalk is a major determinant for dual-task costs in crossmodal response demands. However, since we did not experimentally manipulate temporal task overlap in the previous study, we were not able to determine to what extent a central bottleneck contributed to the observed performance costs. The present study explicitly examined this issue of serial versus parallel processing in crossmodal action selection, complementing, on the action side, an already existing line of research on crossmodal attention in perceptual processing (e.g., Spence \& Driver, 2004).

\section{METHOD}

\section{Participants}

Twenty-four students from RWTH Aachen University with normal or corrected-to-normal vision took part in this experiment and were randomly assigned to either the small temporal task overlap group ( 9 of them female, mean age $=25$ years) or the large temporal task overlap group ( 8 of them female, mean age $=26$ years). They gave informed consent and received credits for participation.

\section{Apparatus and Stimuli}

The participants were seated using a chinrest that was $67 \mathrm{~cm}$ in front of a 21-in. cathode-ray monitor (temporal resolution, $100 \mathrm{~Hz}$; spatial resolution, $1,240 \times 1,068$ pixels). The space bar of a keyboard was used during calibration routines. Saccade RTs (defined as the interval between stimulus onset and the initiation of the saccade) were registered using an EyeLink II system (SR Research, Canada) by measuring the position of the right pupil with a temporal resolution of $500 \mathrm{~Hz}$ and a spatial resolution of less than $0.022^{\circ}$.

A green fixation cross at the center of the screen, as well as two green rectangular squares $6^{\circ}$ to the left and right of fixation, remained present throughout. The size of the fixation cross and the rectangular saccade targets was $1 / 3^{\circ}$ each. On the keyboard, two keys (Alt and right Ctrl) were chosen from the bottom key row as response keys. Participants responded with their left and right index fingers. Throughout the experiment, participants wore headphones for the presentation of the auditory stimuli, which consisted of $1000-\mathrm{Hz}$ sine waves presented for $50 \mathrm{msec}$ to either the left or the right ear with an easily audible intensity.

\section{Procedure}

Each trial began with the presentation of the imperative auditory stimulus $(50 \mathrm{msec})$ to either the left or the right ear. Participants in the small temporal overlap group were instructed to respond as fast and accurately as possible by moving their gaze to the spatially compatible square on the screen (saccade task in single-task blocks), by pressing the spatially incompatible key on the keyboard (manual task in single-task blocks), or by doing both (dual-task blocks). In the large temporal overlap group, the spatial mapping of responses to the auditory stimulus was reversed: Participants were instructed to respond by moving their gaze to the spatially incompatible square on the screen (saccade task in single blocks), by pressing the spatially compatible key on the keyboard (manual task in single blocks), or by doing both (dual-task blocks).

In the single manual blocks, participants were asked to remain fixated on the central fixation cross. In the conditions that required saccades (saccade task in single- and dual-task blocks), participants were instructed to return to the central fixation cross after responses. Each participant completed nine blocks consisting of 30 trials each. Within each block, stimuli to the left and right were presented in a randomized sequence with an interstimulus interval of 3,000 msec. Prior to each block, participants underwent a calibration routine. Participants performed 30 practice trials at the beginning that were not further analyzed.

\section{Design}

The variables of response modality (saccade vs. manual response) and task condition (single vs. dual) were manipulated within participants, whereas temporal task overlap (small vs. large) was manipulated between participants. Participants performed three identical sequences of three blocks - namely, saccade response (single task), dual task, and manual response (single task). The order of conditions within each sequence was counterbalanced across participants. As dependent variables, we measured RTs and errors for saccades and manual responses.

\section{RESULTS}

In the small overlap group, we discarded $6.6 \%$ of the trials in the single-task saccade blocks and $9.9 \%$ of the trials in the dual-task blocks because of blinks or calibration problems with the eyetracker. In the single manual blocks, 
$6.6 \%$ of the trials had erroneously executed simultaneous saccades and were excluded.

In the large overlap group, we discarded $4.7 \%$ of the trials in single-task saccade blocks and $8.4 \%$ of the trials in the dual-task blocks because of blinks or calibration problems. In the single-task manual blocks, $5.7 \%$ of the trials had erroneously executed simultaneous saccades and were excluded.

Figure 1A shows the mean RTs for saccades and manual responses in single-task and dual-task conditions for both the small and large overlap groups. A three-way ANOVA revealed a significant main effect of modality $[F(1,22)=$ $\left.111.39, p<.001, \eta_{\mathrm{p}}^{2}=.84\right]$, indicating longer RTs for manual responses $(637 \mathrm{msec})$ than for saccades $(299 \mathrm{msec})$. There was also a significant main effect of task condition $\left[F(1,22)=69.90, p<.001, \eta_{\mathrm{p}}^{2}=.76\right]$, indicating longer RTs in the dual-task condition (557 msec) than in the singletask condition (379 $\mathrm{msec})$, thus showing overall dual-task costs of $178 \mathrm{msec}$. There was no significant main effect of temporal task overlap $[F(1,22)=2.22, p>.10]$.

The interaction of modality and task condition was significant $\left[F(1,22)=28.49, p<.001, \eta_{\mathrm{p}}^{2}=.56\right]$, indicating greater dual-task costs for manual responses $(289 \mathrm{msec})$ than for saccades $(66 \mathrm{msec})$. A separate analysis in both groups showed that dual-task costs were consistently more pronounced in manual responses than in saccades ( $277 \mathrm{vs.}$ $57 \mathrm{msec}$ in the small overlap group and $301 \mathrm{vs.} 76 \mathrm{msec}$ in the large overlap group; both $p \mathrm{~s}<.001$ ).

The interaction of modality and temporal task overlap was significant, too $\left[F(1,22)=20.74, p<.001, \eta_{\mathrm{p}}^{2}=.49\right]$. This interaction indicates that the overall difference between manual and saccade RTs was substantially greater in the small overlap group $(485 \mathrm{msec})$ than in the large overlap group (192 msec). The assimilation of single-task processing speed (i.e., the reduction of the RT difference between manual responses and saccades in single-task conditions in the large overlap group, as compared with that in the small overlap group) amounted to $79 \%$, providing evidence for the effectiveness of our between-participants manipulation.

Most important, overall dual-task costs were virtually the same in both groups $(167 \mathrm{msec}$ in the small overlap group vs. $188 \mathrm{msec}$ in the large overlap group; $F<1$ for the interaction of task condition and temporal task overlap), and the three-way interaction of task condition, modality, and temporal task overlap was not significant $(F<1)$ (see Figure 1).

Because of the pronounced differences in single-task RTs across groups, we additionally computed proportional dual-task costs for each modality and group. Although the amount of proportional dual-task costs was, overall, higher for the manual responses (62\%) than for the saccades $(25 \%)$ $\left[F(1,22)=11.01, p=.003, \eta_{\mathrm{p}}^{2}=.334\right]$, there was neither a significant difference across groups $[F(1,22)=2.58$, $p>.10]$ nor a significant interaction $[F(1,22)=2.04, p>$ .10]. Mean dual-task interresponse intervals were greater in the small overlap group than in the large overlap group $(595$ vs. $305 \mathrm{msec})[t(22)=2.94, p=.008]$.

Regarding errors, we found a significant main effect of modality $\left[F(1,22)=12.56, p=.002, \eta_{\mathfrak{p}}^{2}=.36\right]$, indicating more errors for saccades $(7.4 \%)$ than for manual responses

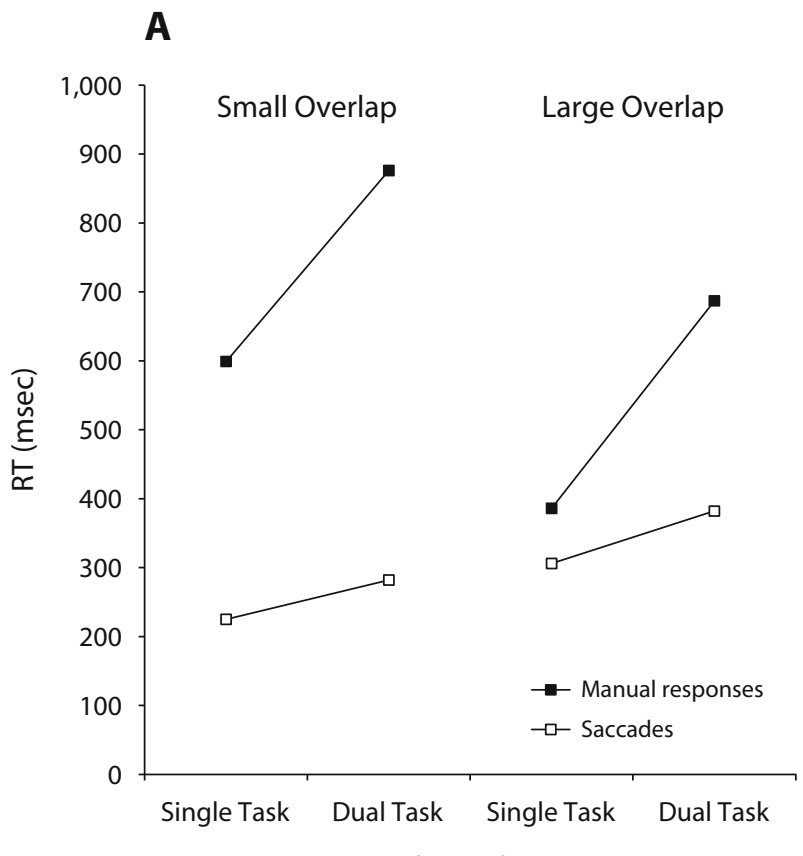

Task Condition

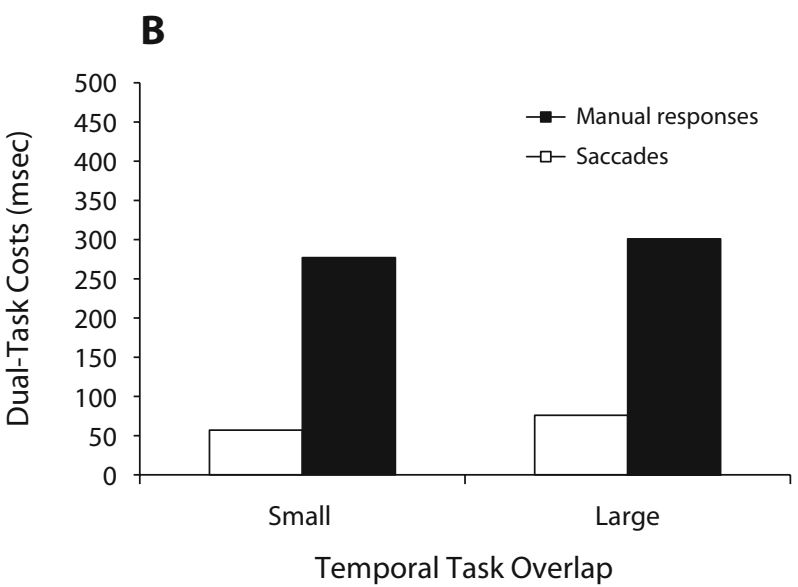

Figure 1. (A) Mean response times (RTs) for saccades and manual responses in single- and dual-task conditions in both temporal overlap groups. (B) Increase of mean RTs in dual- versus singletask conditions (dual-task costs in milliseconds) for saccades and manual responses in both temporal overlap groups.

(2.5\%). Additionally, there was a significant main effect of task condition $\left[F(1,22)=6.45, p=.019, \eta_{\mathrm{p}}^{2}=.23\right]$, indicating more errors in dual-task conditions $(5.9 \%)$ than in single-task conditions (4.0\%). Errors occurred more often in the small overlap group (7.0\%) than in the large overlap group $(2.9 \%)\left[F(1,22)=8.99, p=.007, \eta_{\mathrm{p}}^{2}=.29\right]$, indicating a performance benefit for the latter.

There was no significant interaction of modality and task condition $(F<1)$. The difference in the amount of errors between saccades and manual responses was greater in the small overlap group $(11.2 \%$ vs. $2.8 \%)$ than in the large overlap group (3.5\% vs. $2.2 \%)[F(1,22)=6.51, p=$ $\left..018, \eta_{\mathrm{p}}^{2}=.23\right]$, but there was no significant difference 
in dual-task costs between groups $(F<1)$ nor a significant three-way interaction of task condition, modality, and temporal task overlap $[F(1,22)=1.25, p>.05]$.

The saccade was initiated earlier than the manual response in $98.9 \%$ of the dual-task trials in the small overlap group, as compared with $90.9 \%$ in the large overlap group $[t(22)=2.49, p=.021]$. Eliminating reversals of this sequence did not change the pattern of results, and there was still no significant interaction of task condition and temporal task overlap $(F<1)$ and no significant threeway interaction $(F<1)$.

To see whether the group difference with respect to interresponse intervals was due to the presence of reversals, we additionally compared these intervals after the exclusion of reversals. However, the interresponse interval between saccades and manual responses was still greater in the small overlap group (595 msec) than in the large overlap group (334 msec) $[t(22)=2.87, p=.009]$.

\section{DISCUSSION}

Dual-task costs were present for both response modalities in each of the two temporal task overlap groups, but they were substantially greater for the manual task than for the saccade task throughout, which is in line with an earlier study of simultaneously executed saccades and manual responses (Huestegge \& Koch, 2009). The key finding of the present study was that temporal task overlap did not affect the amount of dual-task costs. We will focus on several potential accounts of this finding below.

\section{Response-Selection Bottleneck?}

The results from the small temporal task overlap group alone are, by and large, consistent with a central responseselection bottleneck framework (Pashler, 1994), since dualtask costs were greater for the slower (manual) task, presumably because response selection in this task has to wait until response selection in the saccade task has been completed. However, enhancing temporal task overlap in the large overlap group should then increase temporal overlap of the central processing stages in both tasks, which, similar to PRP logic, should severely increase dual-task costs. This prediction of the central bottleneck framework was clearly ruled out, since, despite the substantial assimilation of single-task processing speed of $79 \%$, dual-task costs were not significantly affected overall, nor for any of the two response modalities. Instead, participants made even fewer errors in the large overlap group, indicating a performance advantage.

In line with this reasoning, the notion of single-task processing speed difference as a determinant of dual-task cost asymmetry (Posner \& Snyder, 1975) can also safely be rejected (see also Dunbar \& MacLeod, 1984). Finally, it is important to note that the amount of dual-task costs for the manual task is in the range of the whole RTs in the saccade task and cannot therefore be explained as being caused solely by a postponement in the order of one single stage of saccade processing.

A closer look at the RT data allows for a more in-depth evaluation of several possible predictions of the serial bottleneck model. In the dual-task condition of the small tem- poral overlap group, saccades are usually processed first, whereas manual responses are selected afterward, given the substantial single-task RT advantage of $375 \mathrm{msec}$ for the saccades and the finding that saccades were executed first in almost $99 \%$ of the trials. If we assume that this overall processing sequence mainly remains unchanged in the large overlap group as well (given that saccades were still executed first in over $90 \%$ of the trials), the bottleneck model would predict a strong increase of manual RTs, resulting from the increased overlap of response-selection stages. However, if we assume that, in the large temporal task overlap group, manual responses are executed first in at least a portion of the trials, this should be reflected in an increase of dual-task costs for the saccades. However, since we did not observe a substantial effect of temporal task overlap on manual costs or on saccade costs - but, instead, even a decrease of errors in the large temporal task overlap group - neither prediction derived from the bottleneck model was supported by our data.

On a more general level, one might question whether the compatibility manipulation in the present study affects response selection in the same way as compatibility usually affects response selection in the PRP paradigm. The major characteristic of the present study is that participants made two responses to the same spatial attribute of a single stimulus. Previous studies provided evidence for the applicability of the traditional response-selection bottleneck model under these conditions (e.g., Fagot \& Pashler, 1992), and one may speculate that the specific compatibility manipulation in the present study (i.e., triggering two incompatible responses with one stimulus) is, to a substantial degree, similar to the more traditional situation, in which incompatible responses are triggered by different stimuli (see Pashler, 1994). However, it is difficult to draw any final conclusions without specific theoretical alternatives. In the following, we discuss some prominent variants of the bottleneck account and some alternative mechanisms of response selection in the present paradigm.

\section{Latent Bottleneck?}

A typical alternative explanation for not finding increased dual-task costs despite larger temporal task overlap refers to the possibility of a latent bottleneck (e.g., Ruthruff, Johnston, Van Selst, Whitsell, \& Remington, 2003). The notion of a latent bottleneck refers to the assumption that response-selection stages in both tasks do not overlap because selection in the first task is already completed before the second task is ready to enter that stage, rendering any postponement unnecessary. The observed dual-task costs in each temporal overlap group would then be attributed solely to other sources of dualtask costs - for example, crosstalk effects and/or general concurrence costs (e.g., due to expectations; see Gottsdanker, 1979). However, within the bottleneck framework, it remains difficult to explain why dual-task costs were also present for the much faster saccades in the small overlap group, although response selection in the saccade task should hardly ever be postponed, given the substantial single-task RT advantage of 375 msec. Such a backward 
crosstalk effect (e.g., Miller, 2006) is not in line with the assumption of strict seriality in central task processing but rather implies that both responses are processed in parallel to some extent, allowing for mutual interference, presumably as a result of crosstalk between conflicting response codes. However, it should be noted that backward crosstalk effects might still be considered compatible with a more elaborated response-selection bottleneck account when one assumes that, in dual-task blocks, the maintenance of two mapping rules in working memory might slow down response selection for the first response, due to uncertainty about which mapping rule to apply (see Duncan, 1979; Proctor \& Vu, 2009).

Finally, it appears quite likely that the substantial assimilation of single-task processing speed (of 79\%) in the condition with larger temporal task overlap should result in an overlap of response-selection stages in at least a portion of dual-task trials, even when one assumes that the duration of response selection in the present paradigm is comparatively short. Thus, the effectiveness of the present manipulation renders a latent bottleneck account of the present data unlikely.

\section{Competition for Entering the Bottleneck?}

One could further object that, even despite the substantial difference in single-task processing speed in the small temporal task overlap group, the common perceptual processing stage for both tasks could still lead to an equal probability for both tasks to enter the central responseselection bottleneck stage first. This would imply that, in some trials, response selection is carried out for the manual task first, explaining dual-task costs for the saccades.

Figure 2 presents a simplified conceptualization of a bottleneck model, in which both tasks compete for entering the bottleneck stage. Because of the single-task RT differences between compatible saccades and compatible manual responses, the manual response-execution stage generally equals two units instead of only one. The upper part of Figure 2 depicts performance under small temporal task overlap, whereas the lower part depicts performance under larger temporal task overlap. The right column depicts mean overall costs (in units) predicted by the model, assuming equal (50\%) entry probabilities for both central bottleneck response-selection stages. These assumptions imply that, in the small temporal task overlap group, mean dual-task costs should be greater for saccades than for manual responses, since, in half of the trials, the saccade task has to wait until the relatively long manual responseselection stage is completed. However, this prediction is clearly incompatible with the present data.

Alternatively, one might assume different probabilities for entering the response-selection stage, even though perceptual processing is the same for both tasks and response-selection processes are usually considered to be amodal (e.g., Marois \& Ivanoff, 2005; but see Hazeltine et al., 2006). Evidence for this assumption might be derived from the data pattern in the small temporal task overlap group: The substantial single-task RT advantage of $375 \mathrm{msec}$ suggests that response selection in the saccade task should hardly ever be postponed. However, the model in Figure 2 would (for any given values of entry probabilities) still predict a strong interaction between temporal task overlap and effector modality, which we did not observe. Taken together, competition for entering the central bottleneck does not represent a viable explanatory alternative.

\section{Conjoint Responding?}

One might further argue that the single-onset paradigm encourages participants to treat the dual-task requirement

\section{Single-Task \\ Processing Speed

\author{
Dual-Task Processing \\ (RS Bottleneck): \\ Saccades First (50\%)
}

Small Overlap

Dual-Task Processing (RS Bottleneck):

Manual First (50\%)
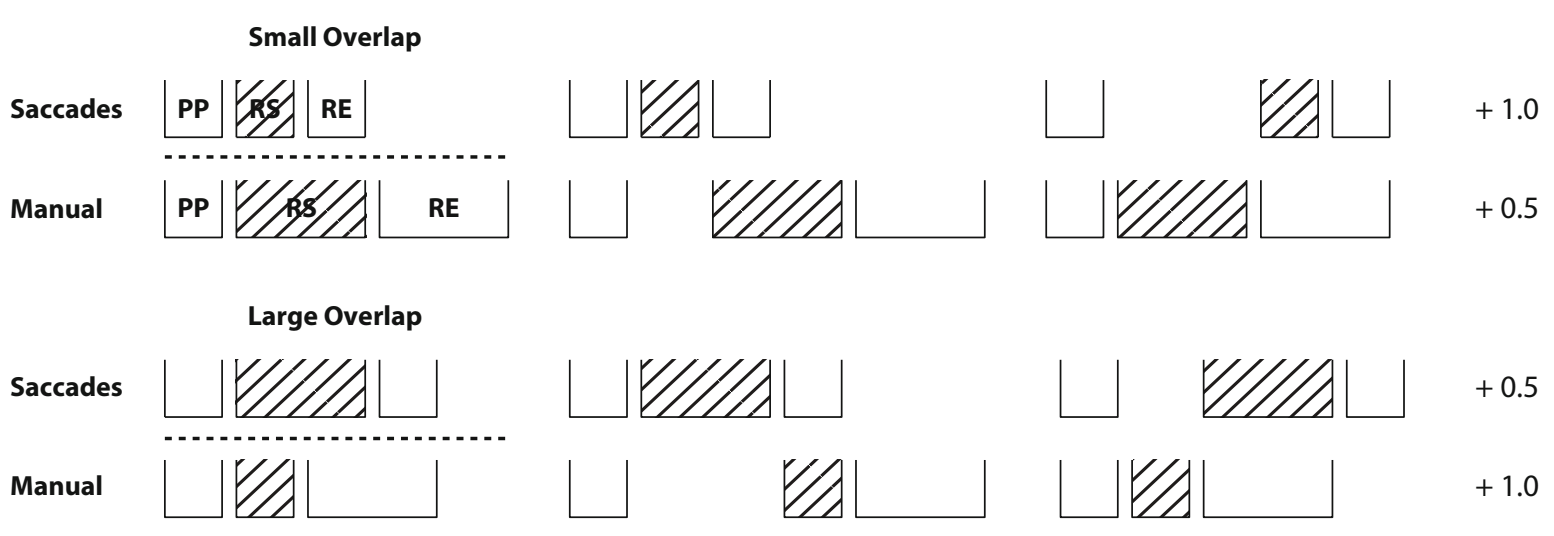

\section{Mean \\ Costs \\ (Units)}

Figure 2. Simplified conceptualization of a central response-selection bottleneck model for the simultaneous performance of saccades and manual responses in the present paradigm. Information processing in each task consists of three stages: perceptual processing (PP), response selection (RS), and response execution (RE). Since single-task RTs were generally prolonged for manual responses versus saccades under otherwise comparable conditions, we assumed a constantly longer $R E$ stage for manual responses (two units instead of one). Note that, regarding dual-task costs, the model predicts a strong interaction of temporal task overlap and effector modality for any given entry probabilities. 
as a single task, basically executing a conjoint response or a single response compound (e.g., Fagot \& Pashler, 1992; Hazeltine, Teague, \& Ivry, 2002). Alternatively, participants might use the direction of the first response as a cue to program the second response, so that the second response is triggered not by the stimulus but by the first response. This should result in a constant interresponse interval, because, under these conditions, the response selection always consists of choosing the opposite spatial code for the execution of the slower-in this case, the manual-response. However, for the following reasons, these arguments seem implausible. First, in a previous study (Huestegge \& Koch, 2009), using a similar experimental setup we found no empirical support for a grouping of manual and saccade responses. For example, even in the simultaneous execution of spatially compatible saccades and manual responses, the interresponse interval was not constant across the distribution of RTs in the first (saccade) task. Second, the present results suggest that the interresponse interval varied substantially across the temporal task overlap conditions (even after removing reversal trials, in which the manual response was executed first), which is incompatible with the claim that responses are grouped (see Ulrich \& Miller, 2008) and the claim that the first response is used as a trigger for the second response (see above). Taken together, these observations render it unlikely that participants treat the dual-task requirement as a single task.

\section{A Parallel Crosstalk Model of Crossmodal Action}

Instead of a central bottleneck, the present findings suggest parallel response-related processing of both tasks, in line with recent accounts of capacity sharing in dual-task control (Meyer \& Kieras, 1997; Navon \& Miller, 2002; Tombu \& Jolicœur, 2003). Navon and Miller (2002) assume that, under certain conditions, the allocation of attentional resources across tasks can be uneven. For example, the sequential presentation of stimuli in the PRP paradigm encourages participants to focus primarily on the first task, which leads to the typical increase of RTs in the second task with increasing task overlap. Meyer and Kieras also argue for a simultaneous application of sets of production rules at the cognitive level, and any asymmetries in dual-task costs may arise as a function of a flexible scheduling of resources. Since both responses in the present experiments relied on common input, central capacity should mainly have been engaged with the resolution of crosstalk between conflicting response codes across tasks (Huestegge \& Koch, 2009; Koch, 2009). Note that, in the present paradigm, the overall cross-response conflict across tasks (in terms of spatial incompatibility between responses) remained constant for both temporal overlap groups, since the same change of the mapping rule was applied to each of the two response modalities.

Figure 3 presents a model of crossmodal action in the present paradigm. It consists of three stages-namely, perceptual processing, parallel mapping selection (as opposed to individual serial response selection in the bottleneck framework), and response execution. Perceptual processing refers to either a left or a right tone, whereas response execution is related to two pairs of responses: either the combination of a leftward saccade and a rightward manual response or vice versa. The pairing of these responses is operated by a binding mechanism in working memory (e.g., Oberauer, 2005), which is indicated by the curved bridge between the two solid vertical lines in Figure 3. The central working memory stage specifies the cognitive mechanism for parallel response selection, which we refer to as mapping selection. The notion of mapping selection instead of individual response selection for each of the two responses (i.e., the central bottleneck assumption) accounts for parallel processing of both tasks, as indicated by mutual interference between both responses (see Duncan, 1979; Proctor \& Vu, 2009). More specifically, amodal spatial codes (left vs. right) need to be bound to corresponding modality codes (saccade vs. manual). The notion of modality codes resembles the concept of separate modalities of information encoding proposed in resource theories of dual-task performance (e.g., Hancock, Oron-Gilad, \& Szalma, 2007; Wickens, 1984). All relevant codes and mappings are assumed to be held active to some extent throughout the experiment, whereas the activation of one of the two alternative mappings (solid vs. dotted lines, referring to the specific mapping triggered by a left vs. a right stimulus, respectively) is boosted on a trial-by-trial basis by the stimulus, resulting in the activation of the corresponding response pair. Since both mappings are randomly intermixed within blocks, crosstalk (indicated by the lightning bolt) in a given trial occurs as a result of persisting activation of the other mapping from previous trials (indicated by the dotted lines) or, alternatively, as a result of the activation of the other mapping as a potential response alternative during the instruction of the participants. Any residual activation of a conflicting mapping subsequently slows down the activation of the correct mapping in the present trial, causing crosstalk. Crucially, crosstalk is affected only by the conflict between mappings, which remained constant across both temporal overlap groups, and not by the specific mapping within each individual group, thus explaining the lack of any substantial alteration of dual-task costs.

On the basis of the present data, we do not wish to claim that central processing stages are conducted in parallel in any given situation regardless of the specific task demands. Rather, we suggest that, although the human information processing system is principally capable of central parallel processing, specific task demands (like those in the PRP paradigm) might lead to a strategic serial processing mode. Furthermore, whenever central processing occurs in parallel, any increase of task demands or between-task conflict may result in a slowing of the speed of processing in either one or both of the individual tasks.

\section{Are Eye Movements Special?}

One might wonder whether saccades are special, in the sense that they bypass central processing stages due to the nature of this specific response modality. Support for this claim comes from previous multitasking research on 

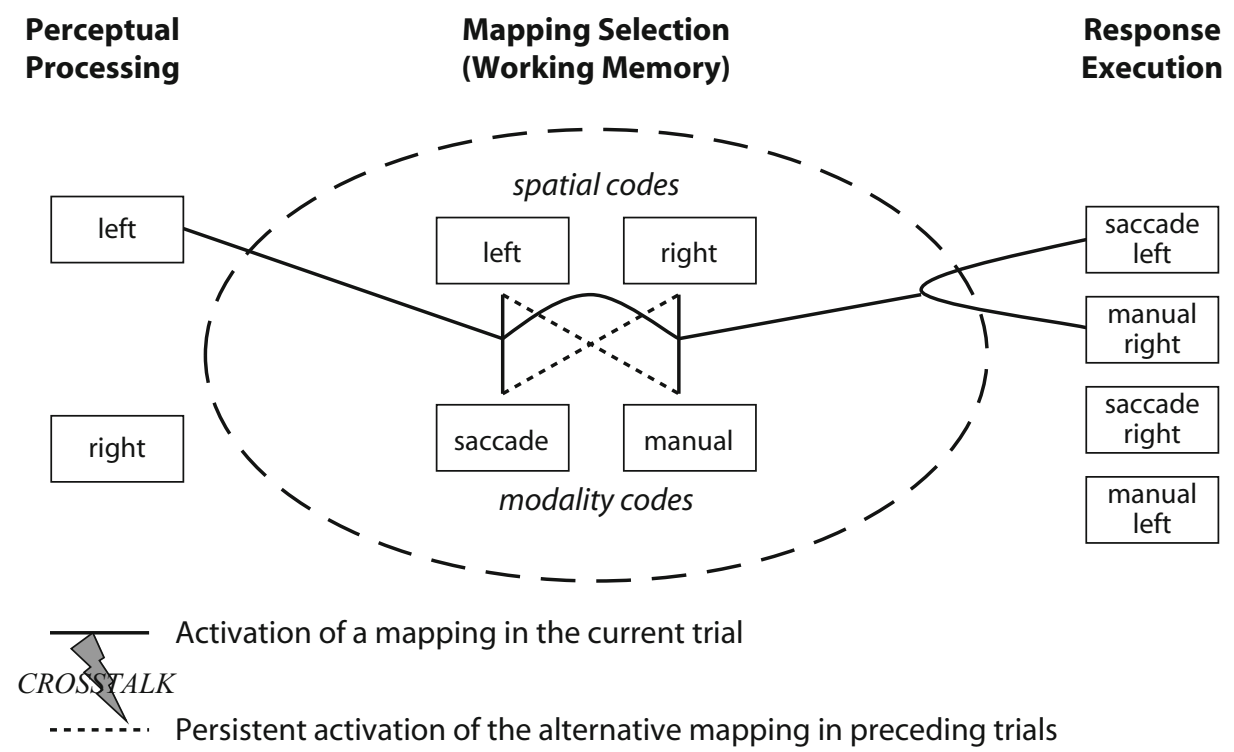

\begin{abstract}
Figure 3. A crosstalk model for the simultaneous performance of saccades and manual responses. Participants respond to a left stimulus with a leftward saccade and a right manual keypress in the current trial. The curved bridges indicate the binding of response pairs in working memory. Crosstalk in dual-task blocks results from interference (the lightning bolt) from alternative stimulus-response patterns of preceding trials (indicated by the dotted lines activated by a stimulus on the right). Additionally, prolonged response times in dual-task trials versus single-task trials may result from the fact that, in dual-task trials, both spatial codes compete for being mapped to modality codes, whereas in single-task blocks, only one spatial code is active, eliminating the potential for competition. Note that, in this model, the specific mapping of spatial codes to modality codes does not affect the general crosstalk mechanism, as long as the (spatial) conflict between responses is the same. Thus, a reversal of the mappings (affecting temporal task overlap) should not alter dual-task costs.
\end{abstract}

saccades and manual responses, which failed to demonstrate any reliable dual-task costs (e.g., Bekkering, Adam, Kingma, Huson, \& Whiting, 1994; Pashler et al., 1993). However, these studies represent a special case, in that they utilized visual stimuli for the saccade task, so that respective responses resemble a quasireflexive orienting response that might be executed with no or negligible central resources. In contrast, in a recent study that also utilized the single-onset paradigm in the study of simultaneously executed saccades and manual responses toward auditory stimuli, we demonstrated that substantial dual-task costs occurred even when both responses were spatially compatible (Huestegge \& Koch, 2009), suggesting that the combination of auditory stimulation and oculomotor responses do not represent a special case that bypasses central processing. Since simultaneously executed saccades and manual responses exhibit the same crosstalk phenomena as do other combinations of response modalities (Huestegge \& Koch, 2009; Navon \& Miller, 1987), it appears likely that further research will provide direct evidence for similar mechanisms in other domains of crossmodal action.

\section{Conclusion}

In the present study, we manipulated temporal task overlap of simultaneously performed saccade and manual response tasks in order to examine corresponding effects on dual-task costs. In sum, the manipulation of temporal task overlap did not affect dual-task costs, as predicted by a serial central bottleneck framework (Byrne \& Anderson,
2001; Pashler, 1994; Salvucci \& Taatgen, 2008), at least not in the present single-onset paradigm. In contrast, our data suggest that central resources are shared in parallel across simultaneously processed saccades and manual responses. Despite the fact that the amount of dual-task costs differed quite substantially between types of responses, they appeared to be equally prone to interference from a temporal prolongation of response selection in the other task, suggesting that crossmodal action is processed in parallel by activating specific mappings between spatial codes and modality codes in working memory, eventually causing response-related crosstalk (Huestegge \& Koch, 2009). Whereas previous multitasking research mainly manipulated temporal task overlap within the PRP paradigm, the combination of the single-onset paradigm with the inverted response incompatibility paradigm provides a novel possibility for studying mechanisms of crossmodal action with suitable control over stimulus-related processes, complementing, on the action side, an already existing stream of research on crossmodal attention in perceptual processing (e.g., Spence \& Driver, 2004).

\section{AUTHOR NOTE}

We thank Elena Zettelmeyer for the collection of the data, and those who kindly volunteered to participate in the study. We further thank Erik Altmann, Robert Proctor, and two anonymous reviewers for their comments on earlier drafts of the manuscript. Correspondence concerning this article should be addressed to L. Huestegge, Institute of Psychology, RWTH Aachen University, Jaegerstrasse 17-19, D-52056 Aachen, Germany (e-mail: lynn.huestegge@psych.rwth-aachen.de). 


\section{REFERENCES}

Bekkering, H., Adam, J. J., Kingma, H., Huson, A., \& Whiting, H. T. A. (1994). Reaction time latencies of eye and hand movements in single- and dual-task conditions. Experimental Brain Research, 97, 471-476.

Byrne, M. D., \& Anderson, J. R. (2001). Serial modules in parallel: The psychological refractory period and perfect time-sharing. Psychological Review, 108, 847-869.

Dunbar, K., \& MacLeod, C. M. (1984). A horse race of a different color: Stroop interference patterns with transformed words. Journal of Experimental Psychology: Human Perception \& Performance, 10, 622-639.

Duncan, J. (1979). Divided attention: The whole is more than the sum of its parts. Journal of Experimental Psychology: Human Perception \& Performance, 5, 216-228.

FAGOT, C., \& PAShler, H. (1992). Making two responses to a single object: Implications for the central attentional bottleneck. Journal of Experimental Psychology: Human Perception \& Performance, 18, 1058-1079.

GotTSDANKER, R. (1979). A psychological refractory period or an unprepared period? Journal of Experimental Psychology: Human Perception \& Performance, 5, 208-215.

HanCoCK, P. A., ORON-Gilad, T., \& Szalma, J. L. (2007). Elaborations of the multiple-resource theory of attention. In A. F. Kramer, D. A. Wiegmann, \& A. Kirlik (Eds.), Attention: From theory to practice (pp. 45-56). Oxford: Oxford University Press.

Hazeltine, E., Ruthruff, E., \& Remington, R. W. (2006). The role of input and output modality pairings in dual-task performance: Evidence for content-dependent central interference. Cognitive Psychology, 52, 291-345.

Hazeltine, E., Teague, D., \& Ivry, R. B. (2002). Simultaneous dualtask performance reveals parallel response selection after practice. Journal of Experimental Psychology: Human Perception \& Performance, 28, 527-545.

Herman, L. M., \& Kantowitz, B. H. (1970). The psychological refractory period effect: Only half the double-stimulation story? Psychological Bulletin, 73, 74-88.

Hommel, B. (1998). Automatic stimulus-response translation in dualtask performance. Journal of Experimental Psychology: Human Perception \& Performance, 24, 1368-1384.

HuestegGe, L., \& Koch, I. (2009). Dual-task crosstalk between saccades and manual responses. Journal of Experimental Psychology: Human Perception \& Performance, 35, 352-362.

$\mathrm{KocH}$, I. (2009). The role of crosstalk in dual-task performance: Evidence from manipulating response-code overlap. Psychological Research, 73, 417-424.

Kornblum, S., Hasbroucq, T., \& Osman, A. (1990). Dimensional overlap: Cognitive basis for stimulus-response compatibility-A model and taxonomy. Psychological Review, 97, 253-270.

Lien, M.-C., \& Proctor, R. W. (2002). Stimulus-response compatibility and psychological refractory period effects: Implications for response selection. Psychonomic Bulletin \& Review, 9, 212-238.

Logan, G. D., \& Gordon, R. D. (2001). Executive control of visual attention in dual-task situations. Psychological Review, 108, 393-434.

MacLEOD, C. M. (1991). Half a century of research on the Stroop effect: An integrative review. Psychological Bulletin, 109, 163-203.

Marois, R., \& IVANOFF, J. (2005). Capacity limits of information processing in the brain. Trends in Cognitive Sciences, 9, 296-305.

Mather, J. A., \& Fisk, J. D. (1985). Orienting to targets by looking and pointing: Parallels and interactions in ocular and manual performance. Quarterly Journal of Experimental Psychology, 37A, 315-338.
Meyer, D. E., \& Kieras, D. E. (1997). A computational theory of executive cognitive processes and multiple-task performance: Part 1. Basic mechanisms. Psychological Review, 104, 3-65.

Miller, J. (2006). Backward crosstalk effects in psychological refractory period paradigms: Effects of second-task response types on firsttask response latencies. Psychological Research, 70, 484-493.

Navon, D., \& Miller, J. (1987). Role of outcome conflict in dual-task interference. Journal of Experimental Psychology: Human Perception \& Performance, 13, 435-448.

NAVON, D., \& MiLLER, J. (2002). Queuing or sharing? A critical evaluation of the single-bottleneck notion. Cognitive Psychology, 44, 193-251.

OBERAUER, K. (2005). Binding and inhibition in working memory: Individual and age differences in short-term recognition. Journal of Experimental Psychology: General, 134, 368-387.

PASHLER, H. (1984). Processing stages in overlapping tasks: Evidence for a central bottleneck. Journal of Experimental Psychology: Human Perception \& Performance, 10, 358-377.

Pashler, H. (1994). Dual-task interference in simple tasks: Data and theory. Psychological Bulletin, 116, 220-244.

Pashler, H., Carrier, M., \& Hoffman, J. (1993). Saccadic eye movements and dual-task interference. Quarterly Journal of Experimental Psychology, 46A, 51-82.

Posner, M. I., \& SNyder, C. R. R. (1975). Attention and cognitive control. In R. L. Solso (Ed.), Information processing and cognition: The Loyola symposium (pp. 55-85). Hillsdale, NJ: Erlbaum.

Proctor, R. W., \& VU, K.-P. L. (2009). Determinants of the benefit for consistent stimulus-response mappings in dual-task performance of four-choice tasks. Attention, Perception, \& Psychophysics, 71, 734756.

Ruthruff, E., Johnston, J. C., Van Selst, M., Whitsell, S., \& RemINGTON, R. (2003). Vanishing dual-task interference after practice: Has the bottleneck been eliminated or is it merely latent? Journal of Experimental Psychology: Human Perception \& Performance, 29, 280-289.

Salvucci, D. D., \& Taatgen, N. A. (2008). Threaded cognition: An integrated theory of concurrent multitasking. Psychological Review, 115, 101-130.

SANDERS, A. F. (1998). Elements of human performance: Reaction processes and attention in human skill. Mahwah, NJ: Erlbaum.

SPENCE, C., \& Driver, J. (EDs.) (2004). Crossmodal space and crossmodal attention. Oxford: Oxford University Press.

Stroop, J. R. (1935). Studies of interference in serial verbal reactions. Journal of Experimental Psychology, 18, 643-662.

Tombu, M., \& Jolicceur, P. (2003). A central capacity sharing model of dual-task performance. Journal of Experimental Psychology: Human Perception \& Performance, 29, 3-18.

Ulrich, R., \& Miller, J. (2008). Response grouping in the psychological refractory period (PRP) paradigm: Models and contamination effects. Cognitive Psychology, 57, 75-121.

Vu, K.-P. L., \& Proctor, R. W. (2006). Emergent perceptual features in the benefit of consistent stimulus-response mappings on dual-task performance. Psychological Research, 70, 468-483.

WeLford, A. T. (1952). The "psychological refractory period" and the timing of high-speed performance-A review and a theory. British Journal of Psychology, 43, 2-19.

Wickens, C. D. (1984). Processing resources in attention. In R. Parasuraman \& D. R. Davies (Eds.), Varieties of attention (pp. 63-102). Orlando, FL: Academic Press.

(Manuscript received April 20, 2009; revision accepted for publication November 9,2009 .) 